\title{
SYNCHROTRON LIGHT INTERFEROMETER AT JEFFERSON LAB*
}

\author{
P. Chevtsov, R. Hicks, Jefferson Lab, Newport News, VA 23606, USA \\ J.-C. Denard, Synchrotron SOLEIL, 91192 CEDEX, France
}

\section{Abstract}

A synchrotron light interferometer has been built at Jefferson Lab in order to measure small beam sizes below the diffraction limit. The device is non-invasive and can monitor the profile of a few microampere electron beam. It follows the design pioneered by $\mathrm{T}$. Mitsuhashi and is a valuable instrument for the CEBAF accelerator. The structure of the interferometer, the experience gained during its installation, and first beam measurement results are presented. Future applications of this device include precise energy spread monitoring $\left(? 10^{-5}\right)$ which is required by some Hall A nuclear physics experiments.

\section{INTRODUCTION}

By recirculating electrons through its two superconducting linacs up to five times, CEBAF provides nuclear physics experiments at Jefferson Lab with powerful (up to $0.8 \mathrm{MW}$ ) electron beams of high quality. Standard beam energies range from 0.8 to $5.7 \mathrm{GeV}$. The beam intensity ranges for three existing experimental end stations are: 1 to 180 ? A for Hall A, 1 to $30 \mathrm{nA}$ for Hall B, and 0.1 to 180 ?A for Hall C. A growing number of experiments at the end stations have begun to require not only very small transverse beam size (down to 20 ?m) and low energy spread $\left(? 10^{-5}\right)$ but also their continuous monitoring at the critical points of the accelerator. One such critical point in the Hall A beam line is the high dispersion $(\sim 4 \mathrm{~m})$ location 1C12 which is shown in Fig.1. This is the area that is very close to a dipole magnet (a blue element at the central part of Fig.1) with the bending radius of 40 meters.

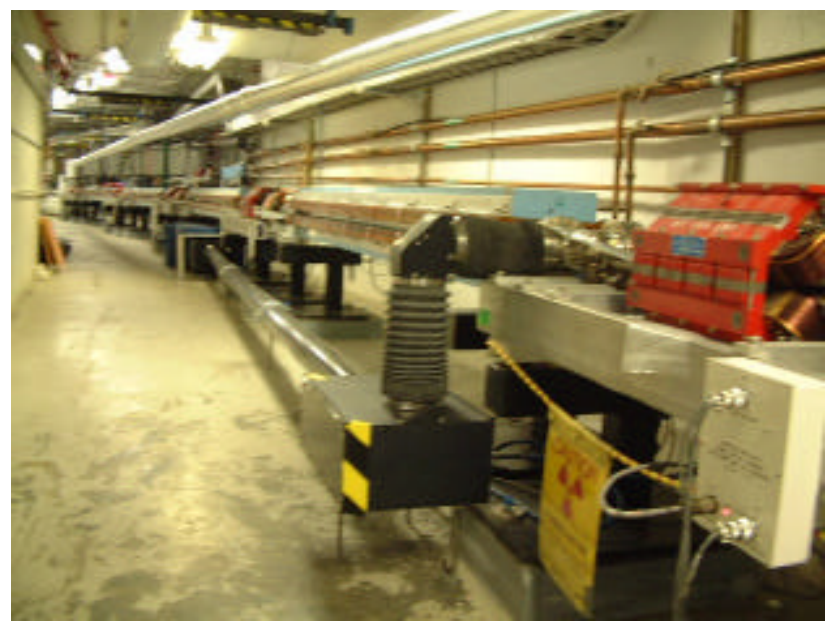

Figure 1: Location $1 \mathrm{C} 12$ in the Hall A beam line with the installed synchrotron light interferometer.

*Supported by DOE Contract \#DE-AC05-84ER40150.
Until recently, location $1 \mathrm{C} 12$ accommodated two beam profile measurement devices. One of them is a wire scanner with 50 ? $\mathrm{m}$ diameter tungsten wires. Beam profiles for low intensities (less than 5 ?A) can be measured once a minute with the accuracy better than 20 ?m. However, such wire scanners are not useful for continuous profile monitoring during beam delivery because they are invasive and cannot withstand the maximum beam current. The second beam profile measurement device at location $1 \mathrm{C} 12$ is an optical transition radiation (OTR) monitor. A very thin $(\sim 0.25$ $? \mathrm{~m})$ carbon foil inserted into the beam path is not invasive for most CEBAF energies and currents. The resolution of this monitor, limited by the used CCD camera to about two pixels, is approximately $60 ? \mathrm{~m}$ of the RMS beam size. This is adequate to the existing but not enough for new beam requirements at Jefferson Lab.

In these conditions, the synchrotron light interferometer (SLI) technique that has been developed at KEK, Japan [1] for measuring very small (down to a few ?m) beam sizes and successfully implemented in several electron storage rings all over the world, was very attractive for a new CEBAF beam diagnostic project. It was decided to build a prototype of a SLI for experimental beam lines at Jefferson Lab as the third beam diagnostic device at location 1C12. The main goals of our synchrotron light interferometer project were to determine the basic structure of the SLI for Jefferson Lab that could easily be replicated and to gain the experience during its installation in the accelerator tunnel and further operations. We also wanted to design and test software that would automate the control functions of all synchrotron light interferometer components and the calculation of the beam size with the use of the SLI data. Three different beam profile monitors at the same location would give us an excellent opportunity to use their measurement results in our work on SLI data processing and analysis models.

\section{SLI AND ITS COMPONENTS}

The first synchrotron light interferometer at Jefferson Lab follows the design pioneered by $\mathrm{T}$. Mitsuhashi [1]. It is a wave front division interferometer that uses polarized quasi-monochromatic synchrotron light. It has a 3-D structure, with major elements placed on two horizontal levels which are parallel to the ground plane (see Fig.1). A very limited space and high radiation level at location $1 \mathrm{C} 12$ were the main reasons to choose and implement this SLI shape.

The synchrotron light generated by the electron beam in the dipole magnet is extracted through a quartz window 
by the mirror installed in a vacuum chamber. After this window, light is shielded by the SLI optical system, until it reaches the CCD video camera connected to the image processor. Two additional adjustable 45 ? mirrors guide light through the optical system. From the first mirror light reflects downward and reaches the second mirror. This mirror is remotely controlled and sends light on the CCD through a long $(\sim 5 \mathrm{~m})$ plastic pipe and all optical components, in the direction opposite to the direction of the electron beam. The CCD and the optical components are placed in an optical box. A diffraction limited doublet lens $(f=1 \mathrm{~m})$ is used as the camera objective. A narrow band optical filter $\left(?_{0}=630 ? 10 \mathrm{~nm}\right)$ is used to obtain a quasi-monochromatic light. A polarization filter selects out the ?-polarized component of the synchrotron radiation. A double slit assembly with different distances between slits (from 5 to $20 \mathrm{~mm}$ ) and small slit openings $(1 \mathrm{~mm} ? 2 \mathrm{~mm})$ is located right in the front of the objective lens. It is moved by a remotely controlled stepper-motor. The SLI optical box is shielded by lead blocks to avoid damage to its components from the high radiation in the accelerator tunnel.

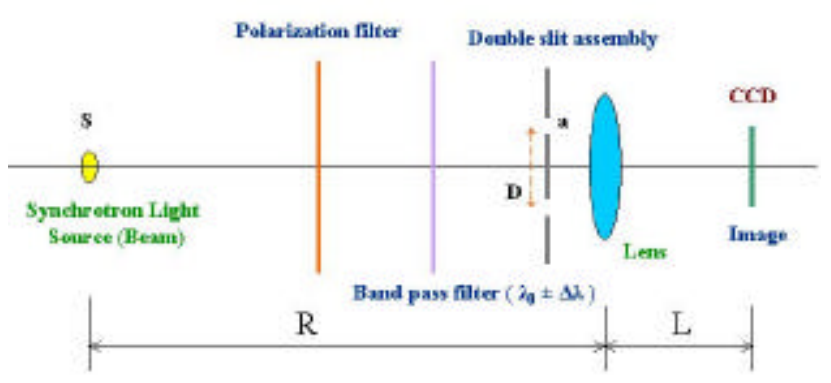

Figure 2: Synchrotron light interferometer outline.

The SLI outline is shown in Fig.2. The double slit assembly is located at the distances R?9.1 $\mathrm{m}$ from the synchrotron light source point and L? $1.1 \mathrm{~m}$ from the CCD.

The SLI video camera is the STV digital integrating video system from the Santa Barbara Instrument Group. The camera has its own control box with the RS-232 interface to an external computer. Its quantum efficiency is very high (more than $70 \%$ ) for $?_{0}=630 \mathrm{~nm}$ and the pixel size is small $(7.4 ? \mathrm{~m} ? 7.4 ? \mathrm{~m})$. An electronic cooling system keeps CCD dark currents extremely low. The exposure time of the camera can gradually be changed from 0.001 seconds to 10 minutes. The SLI image processor is Datacube's MaxVideo MV200 board that is the basic video image processing system for beam diagnostic applications at Jefferson Lab [2]. The STV video camera and MV200 system make it possible for the SLI to measure the sizes of the CEBAF electron beams in a very wide range in energy and intensity.

\section{INTERFEROGRAM ANALYSIS}

The SLI interference pattern is captured by the CCD video camera and analyzed by the image processing software running on the MV200 and its host computer (a Motorola PowerPC based IOC) connected to the accelerator control system based on EPICS. As a part of the distributed real-time EPICS database, the digitized images from the video camera and the information about the calculated beam size are available for the accelerator control computer network and can be used for various beam diagnostic applications.

The basic parameter to calculate the beam size is the visibility $V$ of the interference pattern. The visibility is estimated with the use of the intensities of the first (central) maximum $\left(I_{\max }\right)$ and minima $\left(I_{\min }\right)$ of the interferogram:

$$
V ? \frac{I_{\max } ? I_{\min }}{I_{\max } ? I_{\min }}
$$

Assuming a gaussian beam shape, the RMS beam size can be calculated at the fixed separation of a double slit as [1]:

$$
?_{\text {beam }} ? \frac{?_{0} ? R}{? ? D} \sqrt{0.5 ? \ln ? \frac{?}{? V} ?} ?
$$

where $\mathrm{D}$ is the double slit separation (see Fig.2).

SLI image processing and data analysis are performed by new software that has been developed for this project. The MV200 continually processes a large volume of pixels corresponding to the video frames from the CCD camera using parallel pipeline technology. This technology makes it possible for the MV200 not only to routinely perform such important operations for processing as masking the pixels outside the interferogram region and subtraction of a background image but also to calculate the RMS beam size with the use of equations (1) and (2) at a high rate (up to $10 \mathrm{~Hz}$ in the multiplexed version of the software). The host CPU (IOC) runs a real-time EPICS database with the information about digitized images and calculated beam parameters. It also runs the SLI data analysis software calculating the RMS beam size with necessary corrections due to such effects as the field depth and imbalance between the intensities of the two modes of light illuminating the double slit. The software fits the measured interference pattern using a multi-parameter, non-linear model that is based on the ideas from paper [3].

\section{EXPERIMENTAL RESULTS}

With the configuration of double slits that has been used for the last few months, our synchrotron light interferometer measured the vertical beam size. Hundreds of measurements with different distances between slits have been made and the data of the three beam profile 
monitors at location 1C12 have been analyzed. Typical SLI interferograms are shown in Fig.3.

The measured vertical beam size was in the range from 0.12 to $0.18 \mathrm{~mm}$ for various CEBAF operation modes. The data of all three monitors were in very good agreement with each other.

The most popular beam requirements from the Hall A users are $5 \mathrm{GeV}$ energy and 100 ? A intensity. The SLI beam size measurement time for this accelerator mode and with $\mathrm{D}=5 \mathrm{~mm}$ is about 1 second. Since the amount of the emitted synchrotron light is proportional to the beam energy and intensity, the optimal video camera exposure time for each CEBAF operation mode can be calculated. Our experimental results have confirmed these calculations.
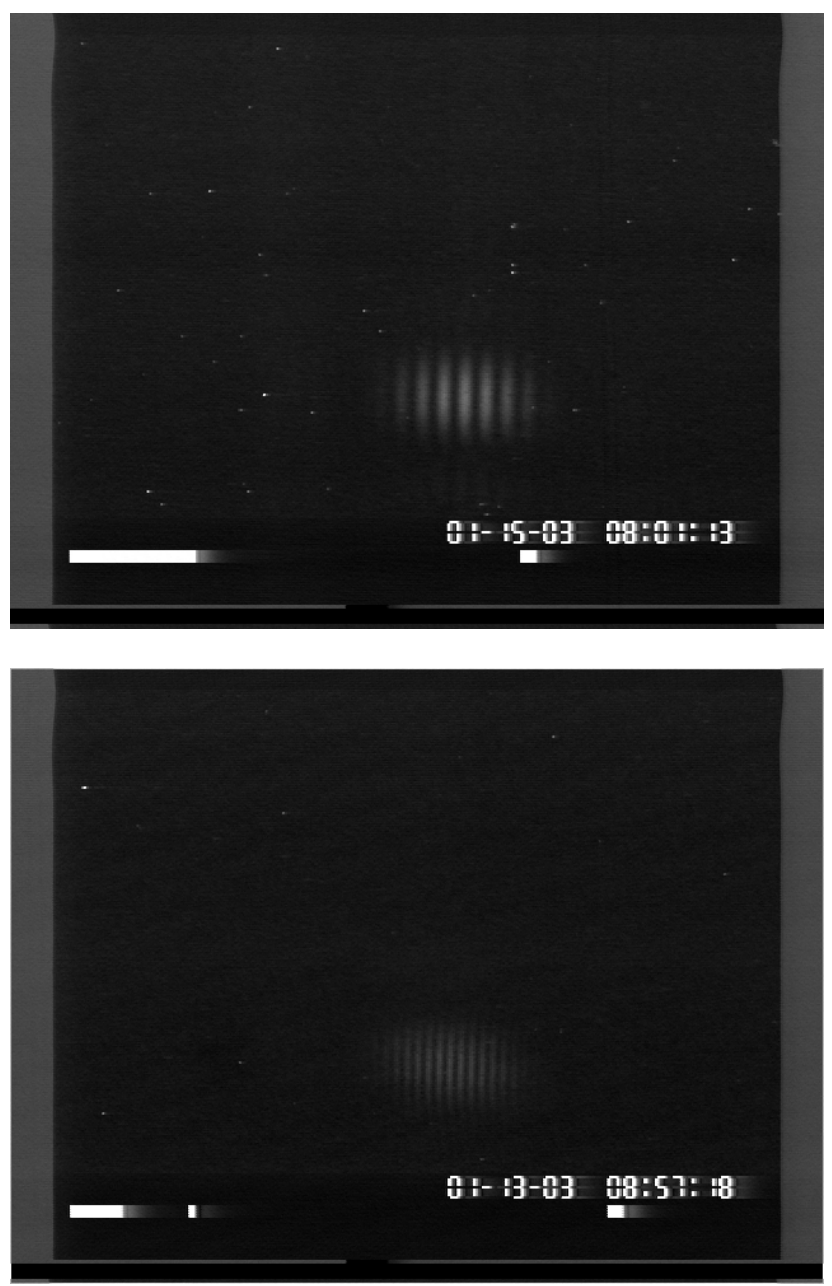

Figure 3. Typical SLI interferograms with $\mathrm{D}=5 \mathrm{~mm}$ (the upper picture) and $\mathrm{D}=10 \mathrm{~mm}$. The calculated beam size is about $0.12 \mathrm{~mm}$ in both cases.

The resolution of the SLI depends on the distance between slits D and can be estimated with the use of equation (2). For example, if $\mathrm{D}=5 \mathrm{~mm}$, then for beam sizes $110 ? \mathrm{~m}$ and $120 ? \mathrm{~m}$ the difference in visibilities is about $3 \%$. Since the image processing system easily calculates the visibility of the CCD image with $1 \%$ accuracy, we can measure the difference between the beam sizes $110 ? \mathrm{~m}$ and $120 ? \mathrm{~m}$ with the resolution better than $10 ? \mathrm{~m}$.

\section{CONCLUSIONS}

The main goals of the SLI project at Jefferson Lab have been achieved. It was demonstrated that the synchrotron light interferometer technique could successfully be used for CEBAF beam diagnostic applications. We have built an automated SLI that is easy to replicate at the other accelerator locations.

The new device is non-invasive and can monitor the profile of a few microampere electron beam. Future applications of this device include precise energy spread monitoring $\left(? 10^{-5}\right)$ that is required by some nuclear physics experiments.

We have gained a great experience in the SLI installation and its further operation. The most difficult problem during the SLI installation in the accelerator tunnel was the alignment of all its optical components around the optical axis formed by the used mirrors. We are planning to implement some modifications in the synchrotron light interferometer structure that would be very helpful for the future synchrotron light interferometer projects. For example, a possibility to use a He-Ne laser as a SLI reference light source should significantly simplify the work on the SLI installation and tests.

\section{ACKNOWLEDGMENTS}

The authors are very thankful to H.Areti, G.Krafft, S. Chattopadhyay, M.Tiefenback, L.Merminga, J.Benesch, A.Bogacz, A.Hutton, K.White, Y.Derbenev, A.Freyberger for their interest in our work and support. The authors also want to thank D. Hardy, S. Suhring, M. Spata, Z. Kursun, A.Day, all accelerator operators and technicians for their help during the SLI installation and tests.

\section{REFERENCES}

[1] T.Mitsuhashi, "Beam Profile Measurement by SR Interferometers", Proceedings of the joint US-CERNJapan-Russia school on Particle Accelerators, Montreux, Switzerland, 1998.

[2] D.Hardy, P.Chevtsov, J.-C.Denard, R.Dickson, "Multivideo Source Image Processing for Beam Profile Monitoring System," Proceedings of Beam Instrumentation Workshop 2000, Cambridge, MA USA, May 2000.

[3] J.Flanagan, S.Hiramatsu, T.Mitsuhashi, "Automatic Continuous Transverse Beam-Size Measurement System for KEKB,' EPAC'2000, Vienna, Austria, 2000. 